\title{
Morphological and electrical characterization of Cu-doped PbS thin films with AFM
}

\author{
Illia Dobryden ${ }^{1,2}$, Baligh Touati ${ }^{3}$, Abdelaziz Gassoumi $^{3,4}$, Alberto Vomiero',Najoua Kamoun ${ }^{3}$, \\ Nils Almqvist ${ }^{1^{*}}$
}

\author{
${ }^{1}$ Department of Engineering Sciences and Mathematics, Division of Materials Science/Experimental Physics, \\ Luleå University of Technology, Luleå, SE-971 87, Sweden \\ ${ }^{2}$ Department of Chemistry, Division of Surface and Corrosion Science, KTH Royal Institute of Technology, Stockholm, \\ SE-100 44, Sweden \\ ${ }^{3}$ Physics Condensed Matter Laboratory, Faculty of Science of Tunis, University of Tunis El-Manar, Tunis, 2092, Tunisia \\ ${ }^{4}$ King Khalid University, College of Science, Physics Department, P.O. Box 9004, Abha 61413, Saudi Arabia
}

*Corresponding author. Tel: (+46) 920492291; E-mail: nils.almqvist@ltu.se

Received: 08 December 2016, Revised: 15 February 2017 and Accepted: 07 April 2017

DOI: $10.5185 /$ amlett.2017.1545

www.vbripress.com/aml

\begin{abstract}
Lead sulphide $(\mathrm{PbS})$ is a direct band gap IV-VI intrinsic p-type semiconductor with good potential for application in solar cells, sensors, etc. Doping the films with $\mathrm{Cu}^{2+}$ ions may improve the electrical properties. Here, $\mathrm{Cu}$-doped $\mathrm{PbS}$ films were deposited on conducting glass substrates. The morphology, topography and thickness of the doped $\mathrm{PbS}$ films were examined using atomic force microscopy (AFM) and high-resolution SEM. AFM analysis showed decreasing surface roughness and grain size with the increase of $\mathrm{Cu}^{2+}$ concentration from 0.5 to 2.0 at\%. Local surface electrical measurements using conducting AFM and Kelvin probe force microscopy showed the possibility to probe semi-quantitatively the changes in surface potential, work function, and Fermi level upon doping of the films. The estimated apparent work function for the undoped $\mathrm{PbS}$ grains in the film was slightly above $4.5 \mathrm{eV}$, while it decreased to a minimum value of 4.43-4.45 eV at $1-1.5$ at $\%$ $\mathrm{Cu}$-doping. Conducting AFM measurements showed that local resistance of the doped samples is lower than on pure $\mathrm{PbS}$ films. These results indicate $\mathrm{Cu}$ doping as an effective strategy to tune the electrical properties of $\mathrm{PbS}$ thin films toward the development of suitable optically active materials for application in photovoltaics. Copyright () 2017 VBRI Press.
\end{abstract}

Keywords: Lead sulphide, KPFM, copper ions, solar cell, thin film.

\section{Introduction}

One of the main tasks in modern world energy development is efficient use of resources and raw materials and development of new high efficiency and promising solutions to exploit solar light. The solutions, on the other hand should be based on low-cost materials possessing outstanding electrical and optical properties to provide good photovoltaic properties. Also, the production of such materials should be based on cheap, clean and energy-efficient technology. For instance, in the case of thin film deposition, the chemical bath deposition (CBD) is a good and efficient option [1]. The IV-VI group semiconducting materials are well-known and studied to be used as photovoltaic material for thin film solar cells. Lead sulphide $(\mathrm{PbS})$ is one of such promising semiconductors, which has a narrow band gap of $0.41 \mathrm{eV}$ at room temperature. Nanocrystalline films of lead sulfide are important for many applications such as solar cells [2] and near infrared optical coatings. Doping can tune the band gap and make smooth films to optimize the layers for solar cell applications (optimized energy gap is 1.34 $\mathrm{eV}$ for absorbing materials in a single junction solar cell, according to the so-called Shockley-Queisser limit [3]). The band gap of $\mathrm{PbS}$ can be varied and it is sensitive to the grain size [4]; the quantum effect in small crystallite sized grains may also alter the optical gap [5]. When $\mathrm{PbS}$ films are used as absorber material in solar cells, the tuning of energy gap, electrical properties and electronic band alignment is important [6]. Doping is an effective route for the purpose $[5,7]$. It is also known that film thickness often influences the measured gap [8]. For PbS films, $\mathrm{Cu}^{2+}$ ions have good potential to substitute $\mathrm{Pb}^{2+}$ in the crystal lattice, and tune the electric properties. Still, there are only a very few studies in literature of the morphological and electrical properties of $\mathrm{PbS}$ thin films doped with $\mathrm{Cd}^{2+}$ or $\mathrm{Cu}^{2+}$ ions [9-11].

Advanced atomic force microscopy (AFM), such as scanning Kelvin probe force microscopy (KPFM) and conductive AFM (C-AFM) methods, are promising for the physical characterization of semiconductor films on a nanometer scale. The KPFM measures contact potential difference (CPD) between a conducting AFM tip and a sample. The effect of doping on the electrical properties and CPD related to the work function, i.e. the minimum required work to remove an electron from within the 
sample to a distance just outside the sample, can be studied with KPFM [12, 13] and is of high interest for various solar cell architectures. However, precautions have to be made. The understanding of the underlying physics and instrumental limitations in the measurements is necessary to extract proper results. The principles and physics have been reviewed by Meritz et al. [14]. The CAFM methods were developed almost 20 years ago for electrical characterization on the nanometer scale [15, 16]. Applying C-AFM and contact mode methods such as scanning spreading resistance (SSRM) to study thin films is a challenging task, but its possibilities of high spatial resolution and local measurements of local surface electrical properties to better understand the electronic transport mechanisms makes it attractive. Ideally, measurements of local spreading resistance can be converted to carrier concentration by using a concentration versus resistivity curve. In the SSRM measurements of a thin film, a constant bias voltage is applied between the conductive AFM tip and the film during AFM contact mode scanning. The contact force is kept large enough to yield a stable electrical contact between the sample and the tip. The resulting local tipsample current and the topography are measured simultaneously, but independently. The measured tipsample current is a result of the applied voltage and the total resistance between the tip and the back of the film where the bias voltage is applied. Hence, the total resistance is the sum of the resistances from the tip, the tip-contact spreading, the sample and the back contact. In many cases, the total resistance is dominated by the tipcontact spreading giving a current that is dominated by the local sample resistivity. The measured currents depend strongly on several factors, including the contact area between the tip and the sample, the loading force, etc., that can vary between measurements. Hence, the quantitative accuracy between different SSRM measurements might be debated. By measuring local currentvoltage (I-V) curves with C-AFM, it is possible to provide information about the transporting mechanisms in localized surfaces areas.

The above mentioned methods, KPFM and SSRM, have lately been applied by others to study photovoltaic thin films, for example the grain boundaries [17]; resistance and non-uniformity from defects [18, 19]; the junction of a CdTe solar cell [20]; $\mathrm{I}-\mathrm{V}$ characteristics of thin films [21, 22]; dopant profiling and carrier concentration [23-26]. In this study we utilized these advanced AFM and HR-SEM methods to characterize the intrinsic $\mathrm{PbS}$ and $\mathrm{Cu}^{-}$doped thin film surface properties. The electrical properties, such as CPD, work function map, spreading resistance maps and I-V curves were measured and analyzed for $\mathrm{PbS}$ films doped with $0.5,1.0,1.5$ and 2.0 at $\% \mathrm{Cu}$. Such characterization of morphological and electrical properties of $\mathrm{Cu}$-doped films at the nanoscale is novel and has, to our knowledge, not been reported by any other group for this system. Moreover, this work proposes a route how to apply these advanced AFM methods to provide valuable information in thin film solar cell analyses at nanoscale at ambient, nitrogen and lowvacuum conditions.

\section{Experimental}

\section{Materials}

Pure and $\mathrm{Cu}$-doped $\mathrm{PbS}$ nanocrystalline thin films were deposited using the chemical bath deposition method on fluorine-doped tin oxide (FTO) glass, which is widely used as a transparent conductor and has high electrical conductivity (FTO sheet resistance being of the order of 8-15 $\Omega / \square)$ and carrier density $[27,28]$. Copper nitrate was added to produce the $\mathrm{Cu}$-doped $\mathrm{PbS}$ films with concentrations $0.5,1.0,1.5$ and 2.0 at $\%$. The deposition was carried out at room temperature during $1 \mathrm{~h}$. The substrates with deposited $\mathrm{PbS}$ films were washed with deionized water and dried in air at room temperature. The exact procedure of the growth of the thin $\mathrm{PbS}$ films is described by Touati et al. [29]. For cross-sectional analysis, the sample was cut from the back of the glass substrate and the freshly cleaved cross-section was studied.

\section{Characterization}

The morphology of the PbS films was examined with a high-resolution SEM (Magellan 400 XHR-SEM). The films were further examined with the AFM-based methods.

\section{Morphological and electrical characterization with atomic force microscopy}

The morphology, topography and electrical properties of the un-doped and copper-doped $\mathrm{PbS}$ films were examined with atomic force microscopy (NT-MDT, NTegra). The AFM was operated in sample scanning configuration with closed-loop scanner position control using capacitive position sensors in all three directions or occasionally with equivalent closed-loop when using smaller range scanners. Tapping mode (semicontact mode) AFM was used for topographic imaging using an n-type silicon NSG01 cantilever (NT-MDT) and the same type of probe coated with a conductive Pt/Ir layer (NSG01-Pt) was used for KPFM and C-AFM. This single crystal silicon probe with resistivity of $0.01-0.025 \Omega \mathrm{cm}$ has a nominal spring constant of $5.1 \mathrm{~N} / \mathrm{m}$. It has a nominal tip curvature radius of $6 \mathrm{~nm}$ and $35 \mathrm{~nm}$ for the un-coated and Pt-coated probe, respectively. The stiff conducting DCP20 probe (NTMDT) with a coating of nitrogen-doped diamond (resistivity $0.5-1 \Omega \mathrm{cm}$, nominal spring constant $48 \mathrm{~N} / \mathrm{m}$ and tip curvature radius of $100 \mathrm{~nm}$ ) and occasionally the Pt-coated multi75E probe (Budgetsensors) were also used for C-AFM. The morphological results presented herein are from AFM imaging performed in ambient air at a relative humidity of $30-40 \%$.

The KPFM measurements were performed with the dual-pass (two-pass) technique using a lift of typically $50 \mathrm{~nm}$ on top of the $\mathrm{PbS}$ layers to minimize the effect of topography on the measured Volta-potentials, while a higher lift, typically $80 \mathrm{~nm}$, was needed on the rougher 
cross-sections. Freshly cleaved highly ordered pyrolytic graphite (HOPG) was used as a reference, with usually determined work function of $4.475 \mathrm{eV}$ [30], to calibrate the measurements. Measurements were done either in ambient conditions or in a dry nitrogen atmosphere with the ground applied to the sample. Since the AFM has a sealed configuration, operation in nitrogen was performed by flowing the whole AFM protective hood with the gas at a slight gauge pressure. If not explicitly written otherwise, the presented KPFM measurements in this paper were acquired in the dry nitrogen atmosphere.

Conductive AFM measurements were done under either ambient conditions or in depressurized atmosphere with the bias voltage applied to the FTO layer contacted with silver paint (Agar Scientific Ltd). The protective AFM hood functioned as the chamber for the low vacuum measurements. The AFM tip and sample was let to sit for some time in the evacuated vacuum chamber. For measurements, vacuum was pumped to typically 0.05 mbar. In our present setup we switch off the vacuum pump and allow the pressure to slowly increase during measurements. The typical pressure for C-AFM measurements was therefore 0.1-1 mbar. If not explicitly written otherwise, the presented C-AFM measurements in this article were acquired in depressurized atmosphere with the NSG01-Pt probe. Simple line-by-line curve and plane fitting was applied to some of the AFM images as a standard procedure.

\section{Results and discussion}

\section{Surface morphology and topography studies}

The high-resolution SEM images (see Fig. S1 in supporting information) for $\mathrm{PbS}$ thin films with $\mathrm{Cu}$ dopant concentration ranging from 0 to 2.0 at $\%$ reveal that the $\mathrm{PbS}$ films are homogeneous and relatively smooth with increase of the concentration up to 2.0 at $\%$ of the dopant. The films are nanocrystalline and the size of the nanocrystallites significantly changes from the pure $\mathrm{PbS}$ film to the $\mathrm{Cu}$-doped $\mathrm{PbS}$ film: The crystallite size decreases with the increase of $\mathrm{Cu}$ ion doping.

To determine and accurately measure the changes in the $\mathrm{PbS}$ crystalline shape and size from doping, AFM measurements were carried out and grain analysis tool was used within the standard AFM Nova (NT-MDT) software. The topographic AFM height images of the $\mathrm{PbS}$ thin films with $\mathrm{Cu}$-dopant concentration ranging from 0 to 2.0 at $\%$ and grain areas defined by the Nova software, are shown in Fig. 1. These images reveal the grains, grain boundaries and crystallite shapes at the different dopant concentrations. It is now clearer than in SEM observation, and can be quantified that the crystalline sizes decrease with the increase in $\mathrm{Cu}$-dopant concentration.

The measurements of average surface roughness and quantitative grain analysis parameters, such as grain length, area and size, of the pure and $\mathrm{Cu}$-doped $\mathrm{PbS}$ films are shown in Fig. 2(a,b). There is a clear difference in an average roughness $(\mathrm{Sa})$ between the pure and doped $\mathrm{PbS}$ films. Moreover, the Sa roughness decreases from around $23 \mathrm{~nm}$ (pure PbS film) to about $9 \mathrm{~nm}$ for the 2 at $\%$ doped film. This can suggest that the optical properties of the $\mathrm{Cu}$-doped $\mathrm{PbS}$ films can be affected and it is known that the film reflection is sensitive to the dopant concentration [31]. Also, the decreasing of grain area and size with increased dopant concentration is clearly observed, as shown in Fig. 2(b). It is also well-known that the grain size affects the energy band gap of $\mathrm{PbS}$ semiconductor and this can alter the electrical properties of the $\mathrm{Cu}$-doped $\mathrm{PbS}$ films.
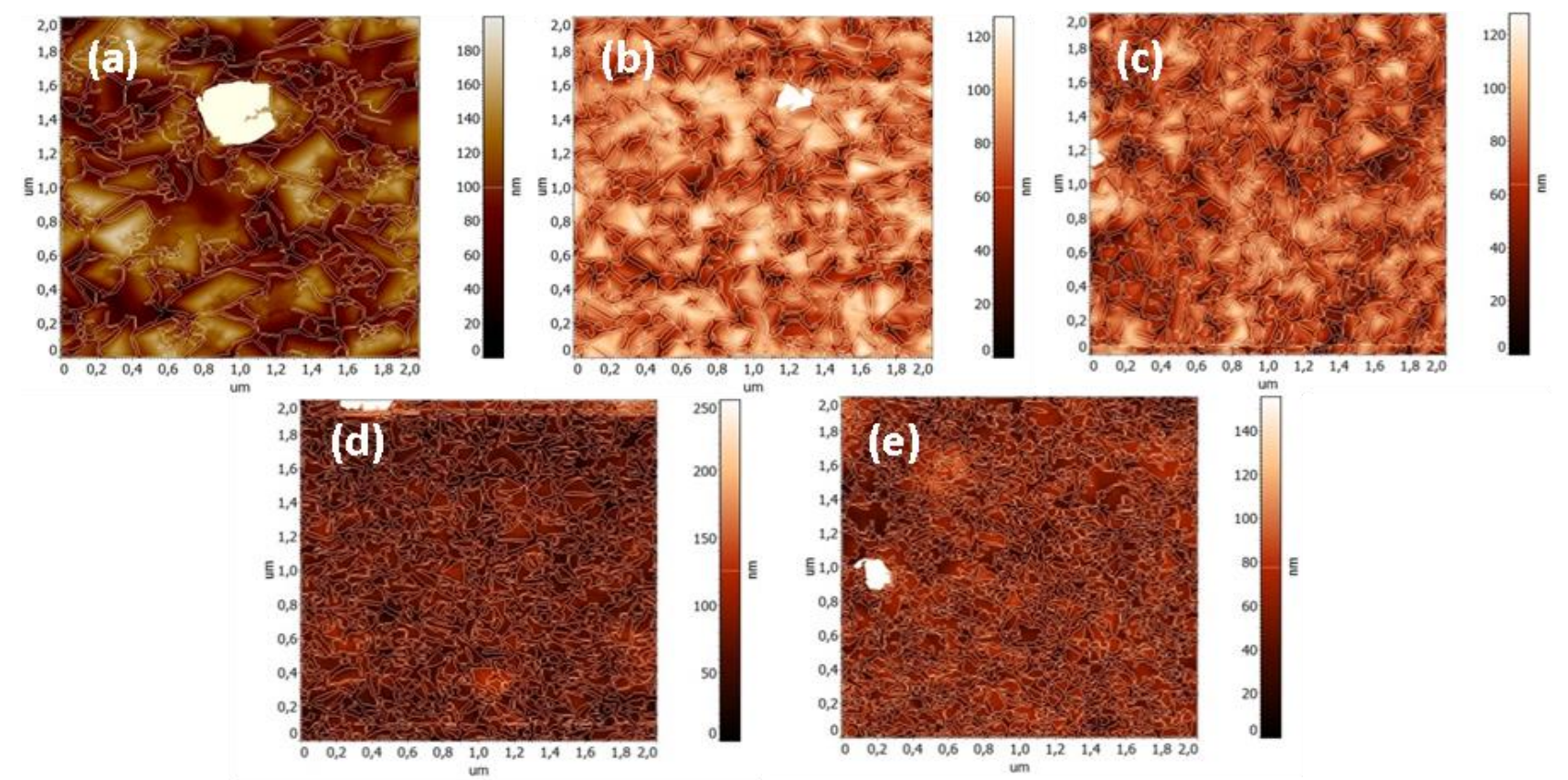

Fig. 1. AFM height images for the pure and $\mathrm{Cu}$-doped $\mathrm{PbS}$ thin films grown on FTO glass by CBD. (a): pure, (b): 0.5 at $\%,(\mathrm{c}): 1.0$ at $\%$, (d): 1.5 at $\%$ and (e): 2.0 at $\%$. The grains are outlined with contours on the images and further analyzed using standard grain analysis tool with the Nova (NT-MDT) software. 

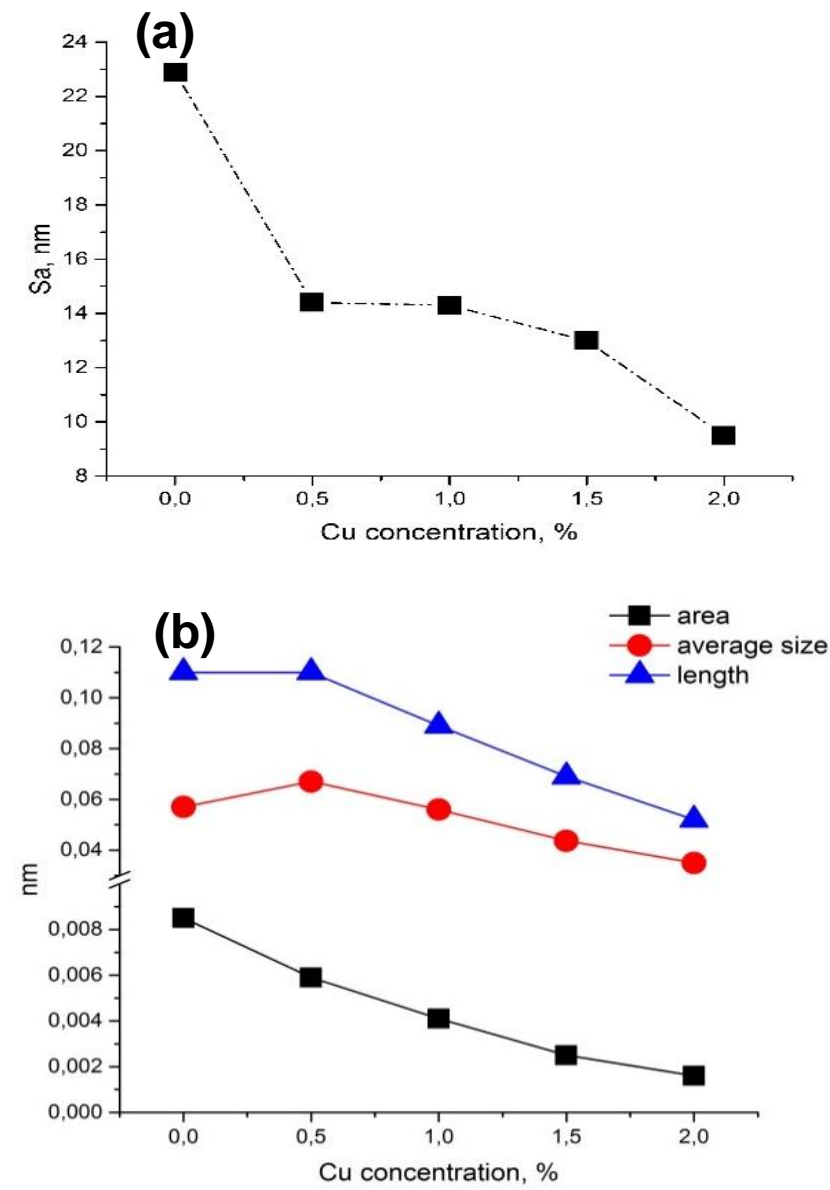

Fig. 2. (a) The evaluated average roughness (Sa) for the pure and $\mathrm{Cu}$ doped $\mathrm{PbS}$ films using AFM; (b) The changes in grain area, average size and length with the increase of $\mathrm{Cu}$ doping.

\section{Local surface potential and potential features}

AFM-based scanning Kelvin probe microscopy (KPFM) was utilized to investigate the effect of Cu-doping, measure surface potential and correlate it with surface morphology and roughness. It should be noted that the detailed evaluation, dopant profiling and modeling of KPFM measurements on semiconductors, especially on p-n junctions, is non-trivial [32]. The contact potential difference measured in KPFM is ideally the position of the Fermi level relative to the AFM probe i.e. here the $\mathrm{Pt}$ or diamond-coated tip. Hence, the KPFM image of the CPD shows the surface charge distribution and variations in electrostatic potential on the sample surface [14]. If the probe work function (WF) is known, it is also possible to estimate the sample WF. The measured CPD is assumed to be directly related to the difference in WF of the tip, $\emptyset_{\text {tip }}$, and the WF of the surface electrons of the sample, $\emptyset_{\text {sample }}$. Hence, $\emptyset_{\text {tip }}$ can be estimated by measuring the CPD on a sample with known WF. As applied by others, freshly cleaved highly ordered pyrolytic graphite (HOPG) is used as the calibration sample. Some research groups prefer to calibrate the CPD to a known material [33]. However, in this work we measured the contact potential difference $C P D_{H O P G}$ on HOPG and used that the work function of the HOPG surface has been determined as $\emptyset_{H O P G}=4.475 \mathrm{eV}[30]$.

Then, the work function of the tip $\emptyset_{\text {tip }}$ was calculated as:

$\emptyset_{t i p}=\emptyset_{H O P G}+|e| C P D_{H O P G}$,

where $e$ is the elementary charge. With known WF of the tip, the contact potential difference on the sample $C P D_{\text {sample }}$, is measured. According to consolidated literature [34], the WF of the sample is estimated as:

$\emptyset_{\text {sample }}=\emptyset_{\text {tip }}-|e| C P D_{\text {sample }}$

We term $\emptyset_{\text {sample }}$ as "apparent WF". A decreasing $\emptyset_{\text {sample }}$ means an increasing CPD since equation 2 shows that the change in apparent WF has different sign as the change in CPD.

The KPFM signal is sensitive to many external sources of noise and imperfections such as contact electrification [35], tip geometry, sample or probe oxidation, adsorption of molecules, humidity, tip-geometry, sample illumination etc. All these can contribute to bad repeatability of measurements especially for measurements in ambient air. In the measurements, it was discovered that $\emptyset_{\text {tip }}$ of the platinum-coated probe often decreased slightly with time as it was used, especially in the ambient measurements. Therefore, it was always important to calibrate the probe's WF on freshly cleaved HOPG both before and after KPFM measurements on a surface. Nevertheless, the interpretation of KPFM results and the effect from CPD on work function is non-trivial. Considerations of band bending and the presence of additional surface charges make even more difficult the detailed evaluation.

The AFM-KPFM images measured in nitrogen atmosphere for pure, 0.5 and 1.0 at $\% \mathrm{Cu}$-doped $\mathrm{PbS}$ film surfaces are shown in Fig. 3 a-f. Some of the images were subjected to a carefully treated line fit in the fast scan direction. The AFM scan velocity in this experiment was $4 \mu \mathrm{m} / \mathrm{s}$ and the measured $\emptyset_{\text {tip }}$ was $4.64-4.65 \mathrm{eV}$. As shown before, the grains/crystallites on the pure sample are larger than on the doped samples and as seen in Fig. 3a. The apparent $\mathrm{WF}$ varies on the pure $\mathrm{PbS}$ within a grain. It is obvious from d-f that the WF of the pure sample $(0$ at $\%)$ is higher (i.e. the CPD lower) than on the $\mathrm{Cu}$-doped samples i.e. there are shifts in the Fermi energies. On the images of $\mathrm{Cu}$-doped samples, almost all the grain surfaces of the same sample have the same work function and only minor lateral differences are detected within each grain. The potential is lower in-between the grains. One should remember that the dual-pass KPFM has higher energy resolution than many other KPFM techniques, but also a lower spatial resolution, though good enough for these conclusions, typically better than $25 \mathrm{~nm}$ [14]. Fig. 3g shows probability histograms of the apparent WF for the pure and the four different $\mathrm{Cu}$-doped $\mathrm{PbS}$ films and Fig. 3h shows the average of these compiled into a graph. The WF is significantly lowered by the $\mathrm{Cu}$-doping. As follows from the definition of WF, 
the electrons are easier extracted from the surface and indicating that the surface is possibly more conductive.

The lowest WF in this experiment is measured on the 1.0 at $\% \mathrm{Cu}$-doped sample in nitrogen atmosphere, and 1.5 at\% Cu-doped sample in ambient conditions. Our measurements yielded WFs as $4.52 \mathrm{eV}$ for the un-doped film and 4.43-4.45 eV for 1.0 at\% doping. As a comparison, classic Kelvin probe measurements on $\mathrm{PbS}$ nanocrystals reported a WF of $4.7 \mathrm{eV}$ [36]. The measurements in ambient conditions show the same overall trend in WF as function of doping as in Fig. 3h, but with slightly lower values and variation between experiments, especially for the two most doped samples. These findings agree well with expectations due to the doping with $\mathrm{Cu}^{2+}$ and with Hall effect measurements reported previously [29], where the resistivity has significantly decreased from pure to $\mathrm{Cu}$-doped $\mathrm{PbS}$. homogenously distributed over the grains. As seen in the figure, there were no sign of current spikes or other effects at the grain boundaries. The measured currents are higher for positive sample bias voltages than for the same negative bias. By extracting the most probable current value from histograms of the images in Fig. 4 it was concluded that the largest increase in conductance, largest for positive bias, appeared at voltages larger than $\pm 1.0-1.5 \mathrm{~V}$. Above this threshold, the film had the typical high resistance of $370 \mathrm{M} \Omega$ (negative bias) and $170 \mathrm{M} \Omega$ (positive bias). When interpreting further results from these measurements, one should keep in mind that the currents are influenced by the total resistance between the AFM tip and the back contact. The metal platinum tip semiconductor sample contact is not necessarily ohmic. Some research groups have recently shown that Pt-coated silicon tips give ohmic contacts on gold and carbon
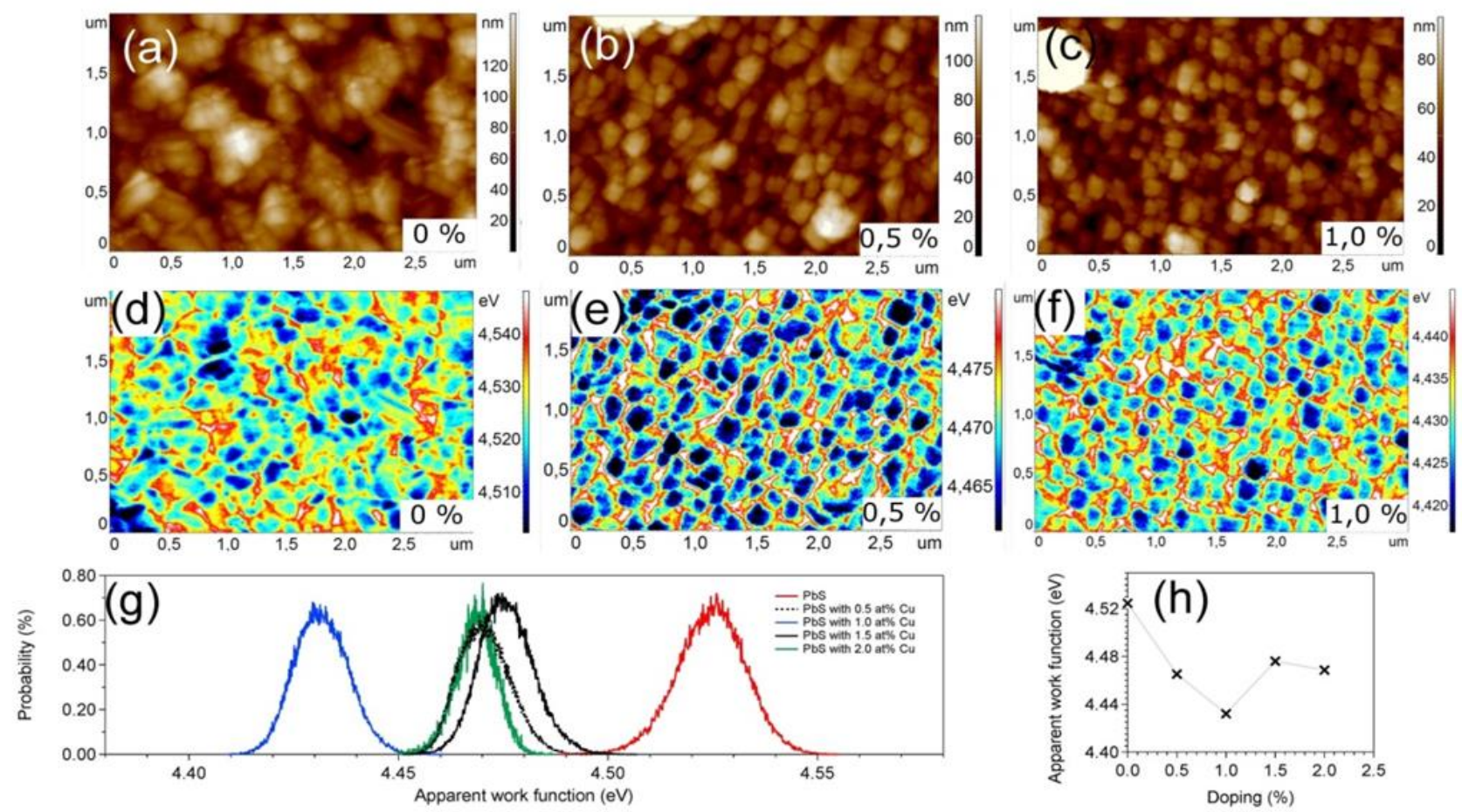

Fig. 3. Results from scanning Kelvin probe microscopy of the samples. The panels (a-c) show the AFM height images of the PbS films at $0,0.5$ and 2.0 at\% Cu-doping. (d-f) is the corresponding apparent WF, i.e. calculated from the measured contact potential difference acquired at a lift of $50 \mathrm{~nm}$, of the same areas as in a-c. The apparent WFs for all the samples are shown in (g) as probability histograms and in (h) as a function of doping. The trend line in $\mathrm{h}$ is just as a guide for the eye.

\section{Local sample currents}

The bias voltage was applied to the FTO layer through the silver paint contact, while the AFM tip was grounded in the C-AFM experiments. All SSRM measurements were performed in ambient air or depressurized atmosphere in the range 0.01-1 mbar. The low-vacuum is supposed to contribute to a more reproducible atmosphere. By pumping vacuum, the amount of contaminating particles/ molecules and moisture is reduced, also minimizing the possible oxidation to the surface. Fig. 4 shows representative two-dimensional SSRM current maps of the 1.0 at\% $\mathrm{Cu}$-doped $\mathrm{PbS}$ film at $0.30 \mu \mathrm{N}$ loading force $(0.35$ $\mu \mathrm{N}$ total tracking force). Prior to imaging the sample and AFM tip was left to sit in pre-pumped vacuum overnight. The SSRM images show that the current is high and nanotube films. More important for consistent results, they have low resistivity, reasonable hardness and small radius of curvature [37]. Still, many of the probe manufactures do not recommend using Pt-coated silicon tips with currents exceeding $10 \mathrm{nA}$. Otherwise, the coating can be damaged at high currents or weared during scanning, making the underlying silicon tip contributing to the current measurement. Imaging the same sample with the doped DCP20 probe at the high loading force of $0.54 \mu \mathrm{N}$ (total tracking force $0.8 \mu \mathrm{N}$ ) yielded higher absolute currents than in Fig. 4 but, also in this case, higher currents for positive than for negative sample bias voltages. The higher current values in this measurement are expected, due to lowered contact resistance at the higher AFM loading force i.e. higher tip-sample contact area. 

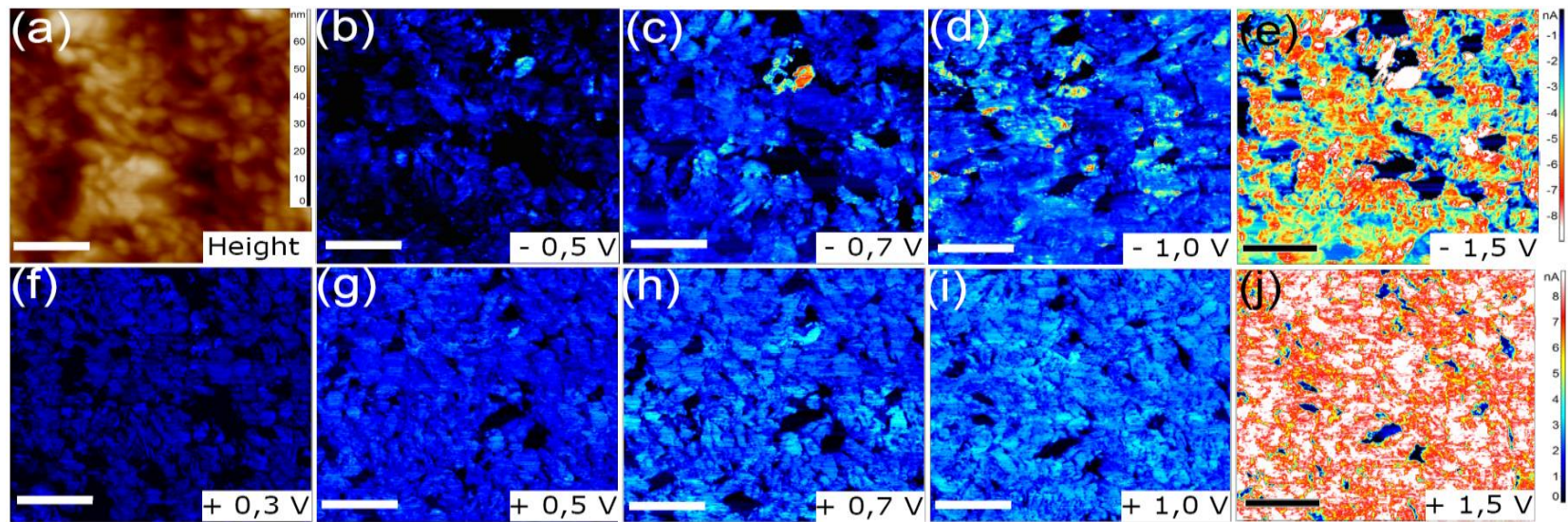

Fig. 4. Scanning spreading resistance images of $\mathrm{PbS}$ films doped with 1.0 at $\% \mathrm{Cu}$ collected at a loading force of approximately $0.30 \mu \mathrm{N}$ : (a) AFM height image of the measured sample area, (b-e) Current images of the same area at sample bias $-0.5,-0.7,-1.0$ and $-1.5 \mathrm{~V}$, (f-j) Current images of the same area at sample bias $+0.3,+0.5,+0.7,+1.0$ and $+1.5 \mathrm{~V}$. The absolute color coding (color bar) of all current images are the same but inverted in the lower row (positive bias). Hence, the same absolute values of the current yields the same color in the image. The scale bars are $500 \mathrm{~nm}$.

The corresponding imaging on the pure $\mathrm{PbS}$ film (data not shown) gave lower currents at negative bias than on positive bias i.e. a rectifying behavior and generally higher resistance than on the 1.0 at $\% \mathrm{Cu}$-doped sample.

These results were further verified by measurements of current-voltage curves (I-V curves) as shown in Fig. 5. During collecting I-V curves the sample bias was swept from a positive to a negative value and then back to the positive value again. We denote this as negative and positive sweep, respectively. I-V curves on surface points that showed hysteresis, i.e. not the same trace on positive and negative sweep, were considered as unstable and rejected. Still such curves could be repeatable for hundreds of curves in the same point. Such hysteretic appearance might be interesting but is out of the scope of this work since it can arise from charging, local heating, break through and from many other effects. Current fluctuations and degeneration have been explained by others to rise from mechanical instabilities, electron tunneling transport and atomic rearrangements at the contact junction [38]. It has already been shown that the conductance between a Pt-coated AFM tip and a gold film is only stable under high contact forces i.e. in the range of $0.5 \mu \mathrm{N}$ [39]. In the current work, we only accounted curves that were repeatable for a large number of sweeps and did not reveal any hysteresis between positive and negative sweep [40].

The curve $g$ in Fig. 5 shows the average of $20 \mathrm{I}-\mathrm{V}$ curves on the same grain surface spot measured with the Pt-coated tip in depressurized atmosphere and is illustrative for measurements on the pure $\mathrm{PbS}$ film. In general, the localized I-V spectra collected on the undoped $\mathrm{PbS}$ surface showed instabilities. Measurements showed the same overall features but differed between different surface points and even show very clear hysteresis. There is a clear asymmetry for positive and negative voltages in the curve with rectifying, Schottky contact-like behavior. As done by others [41], plotting the most probable value of the current from current images of the pure $\mathrm{PbS}$ film acquired at different bias voltages into the same graph (data not shown), give exactly the same rectifying behavior as in curve $g$. From the KPFM measurements, we expect the WF of the Pt-coated tip to be higher, but only slightly, than the pure $\mathrm{PbS}$ surface i.e. $\emptyset_{t i p}>\emptyset_{P b s}$. In the most idealized model of a metalsemiconductor contact, neglecting Fermi level pinning and other surface effects, such contact is supposed to be rectifying when the semiconductor is of n-type and ohmic if it is of p-type (see for example [42]). In a real measurement, as herein, it is not unlikely that the contact is rectifying even if the semiconductor is of p-type. In fact, considering that the bias voltage in our measurement is applied to the sample $\mathrm{PbS}$, the shape of curve $g$ in Fig. 5 might be explained as the typical current-voltage characteristics for a rectifying metal to p-type semiconductor contact forward biased for positive voltages in the graph $[43,44]$.

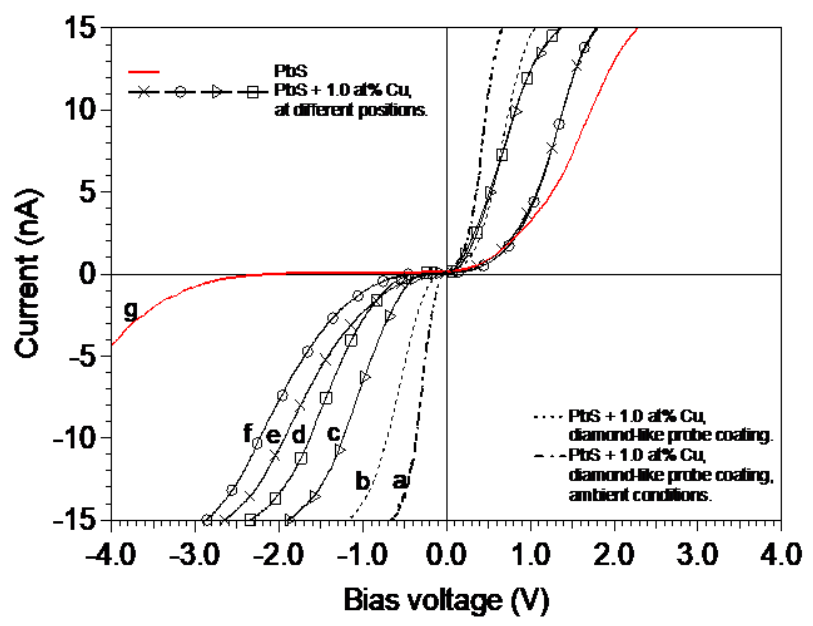

Fig. 5. I-V curves from C-AFM measurements of the pure and the 1.0 at\% $\mathrm{Cu}$-doped $\mathrm{PbS}$ samples. Each displayed curve is the average of 20-100 curves measured in a single surface point. All curves, except $a$, were measured in low vacuum conditions. Curves $a-b$ are measured with the diamond-like carbon-coated tip at a high tip-sample contact force while all others are with the Pt/Ir-coated tip. The curves $c-f$ are from 
different surface points on the doped sample. The curve $g$ is measured on the pure $\mathrm{PbS}$ sample.

The same measurement with the Pt-coated tip on the 1.0 at\% Cu-doped film, Fig. 5(c-f), did not clearly show any rectifying behavior but a localized "band gap" for the current. This electrical "band gap" varies $0.8-1.4 \mathrm{eV}$ on the randomly chosen different locations. It has been shown that the band gap of $\mathrm{PbS}$ thin films increases as film thickness decrease and barrier height increased [45], but our measurements are in the range of what can be expected for the measurements of optical band gap [29].Moreover, plotting values measured from current images at different bias voltages into the same graph (data not shown) fits well with the collected I-V curves of the 1.0 at\% $\mathrm{Cu}$-doped film. Measurements with the diamondcoated tip, curve a-b in Fig. 5, gave an unexpected low "band gap". Since a high tip-sample force was used with the diamond-coated tip, we speculate that this measurement was too harsh to the thin $\mathrm{PbS}$ film and therefore partly probed the underlying FTO layer. The comparison of I-V curves for the pure and doped sample shows that the local resistivity of the doped sample are lower above the cut-in voltage i.e. the slopes of the curves in Fig. 5 are higher. Differently from the curves on pure $\mathrm{PbS}$, the $\mathrm{I}-\mathrm{V}$ curves on $\mathrm{Cu}$-doped films are fairly symmetric on negative and positive bias voltages. As described earlier, the work function of the doped films $\emptyset_{P b S / C u}$ is lower than on the pure sample. Therefore, it clearly holds that $\emptyset_{t i p}>\emptyset_{P b S / C u}$. This might provide a more ohmic contact if the $\mathrm{Cu}$-doped sample is of p-type. The I-V characteristic, more specifically the threshold bias necessary to measure currents, can also depend on the grain size, which is different on the two samples. However, it cannot be out ruled that the rectifying behavior due to imperfections in the measurements such as the n-type silicon tip contributing to the measurement.

\section{Cross-sectional studies}

Of particular interest was also to determine accurately the thickness and electrical properties of the manufactured 1.0 at\% $\mathrm{Cu}$-doped $\mathrm{PbS}$ films. For cross-sectional analysis, the sample was cut from back of the glass substrate and the virgin cross-section surface was studied "as is". The highresolution SEM image and AFM height image of the cross-section region are shown in Fig. 6. The thickness of about $500 \mathrm{~nm}$ for the $\mathrm{F}: \mathrm{SnO}_{2}$ layer and of about $120 \mathrm{~nm}$ for the $\mathrm{PbS}$ layer are measured. The measured thickness of the layers are in agreement with the AFM measurements in Fig. 6 and also agrees with the thickness determined by Rutherford backscattering spectrometry reported in [29]. Moreover, the conducted SSRM measurements in ambient air with the diamond-coated AFM probe on the 1.0 at $\%$ Cu doped PbS sample crosssection, demonstrates a high conductivity for the $\mathrm{F}: \mathrm{SnO}_{2}$ layer which is easily distinguished from the glass surface. It is unclear if the thin $\mathrm{PbS}$ thin film can be measured with SSRM on the cross-section, since these tip outer radius of at least $50 \mathrm{~nm}$ will convolute and overlap with the contribution to the current measurements on $\mathrm{F}: \mathrm{SnO}_{2}$. However, a thin and less conductive layer can be still observed on the edge, which can possibly be regarded as the $\mathrm{PbS}$ layer.

\section{Conclusion}

We report on the morphological and electrical characterization of $\mathrm{Cu}$-doped $\mathrm{PbS}$ thin films using AFMbased methods together with high-resolution scanning electron microscopy. We demonstrate that with some precautions, KPFM and C-AFM can be used to probe surface potential, work function, change in Fermi level, ultimately for density of states and especially changes therein upon doping with copper. Such electrical characterization of $\mathrm{Cu}$-doped $\mathrm{PbS}$ thin films utilizing AFM is reported here for the first time, as for our knowledge. The obtained results can be useful for future development in this field, to optimize the $\mathrm{Cu}$-doped $\mathrm{PbS}$ thin films to increase solar cell photoconversion efficiency.

The thickness of the conductive $\mathrm{F}: \mathrm{SnO}_{2}$ layer was found to be around $500 \mathrm{~nm}$ and the thickness of the $\mathrm{PbS}$ film of around $120 \mathrm{~nm}$ as determined with HR-SEM cross-section analysis of the $1.0 \mathrm{at} \% \mathrm{Cu}^{2+}$ doped film. Copper incorporation in the $\mathrm{PbS}$ films had a strong effect on the surface morphology. The doping resulted in a drastic decrease of average surface roughness, which was compatible with a decrease in nanocrystallite size by about $60 \%$. The crystallite grain shape was triangular and did, however, not change much with the dopant concentration increase from 0.5 to 2.0 at $\%$. The KPFM and C-AFM showed laterally homogenous electrical properties of the crystallites of the doped films. The CPD
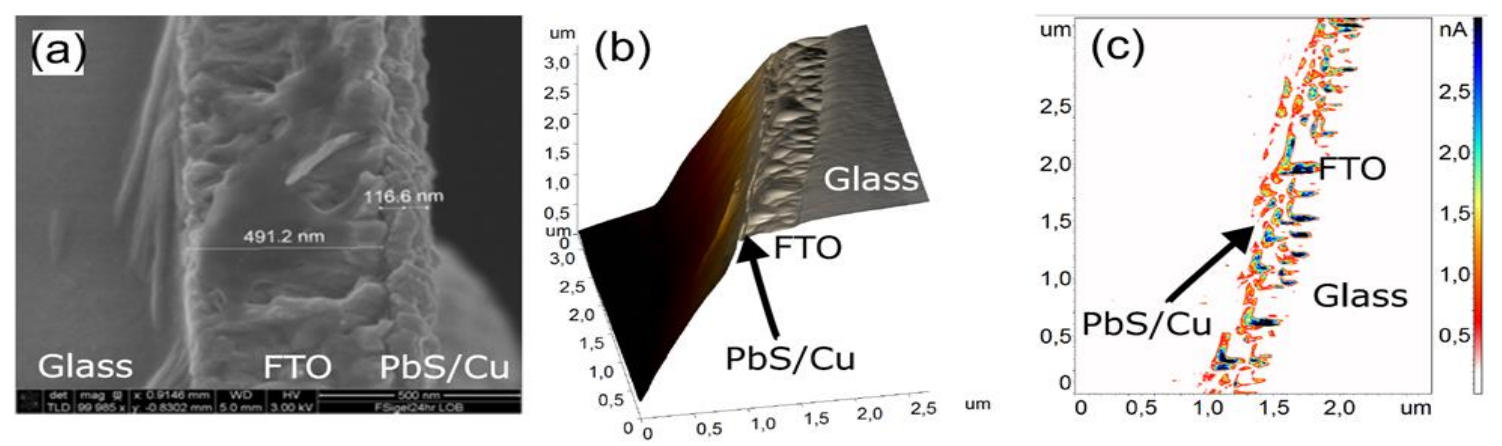

Fig. 6. Cross-sectional study of the 1.0 at $\% \mathrm{Cu}$-doped PbS film. (a) HR-SEM image; (b) Contact mode AFM virtually illuminated 3D height image; (c) SSRM image in ambient air at a sample (FTO) bias of 0.1 volt. 
data from KPFM measurements was used to estimate apparent work function of the crystallites. Measurements in ambient conditions fluctuated but yielded the same overall result on the effect of doping as measurements in nitrogen, i.e. doping decreased the work function (i.e. increased CPD) to a minimum at $1-1.5$ at\% copper. The work function for the un-doped film was $4.52 \mathrm{eV}$, while it was $4.43-4.45 \mathrm{eV}$ for 1.0 at\% doping. The challenging CAFM measurements, SSRM and I-V spectroscopy, complemented these findings by showing that local resistance of the investigated doped samples are lower than on pure $\mathrm{PbS}$ films and indicating their more p-type character. This, together with the morphological studies, confirms earlier findings [29] that copper ions are incorporated into the $\mathrm{PbS}$ crystal lattice, and tune its energy band gap. Also, it demonstrates a correlation between the AFM electrical characterization at the nanoscale and a Hall effect measurement at the macroscale [6, 29], as an increase of film conductivity with $\mathrm{Cu}^{2+}$ doping around 1.0 to $1.5 \mathrm{at} \%$. Measurements with the Pt-coated probe gave a Schottky-type contact on pure $\mathrm{PbS}$, while I-V curves on the doped samples were symmetric. One reason for this might be the difference in work function between these samples. Hence, a local band gap can be roughly estimated on the doped samples as 0.8 $-1.4 \mathrm{eV}$. Regardless of this wide interval, this is higher than expected for the un-doped $\mathrm{PbS}$ and can make the $\mathrm{Cu}$ doped $\mathrm{PbS}$ films attractive as absorber layer in solar cell devices. The AFM methods applied on cross-sections allowed to visualize and separate FTO layer and possibly a thin $\mathrm{PbS}$ film. However, more effort is needed to gain this local electrical data and their proper interpretation.

\section{Acknowledgements}

The Kempe Foundations are highly acknowledged for funding the AFM equipment under the grant SMK-2546. A.V. acknowledge the Swedish Foundations for partial support through a Consolidator Fellowship. Pedram Ghamgosar is thanked for general lab and SEM measurements assistance.

\section{Author's contributions}

Performed the experments: NA, ID, BT, AG, NK, AV; Data analysis: NA; ID; Wrote the paper: NA, ID, AV. Authors have no competing financial interests.

\section{Supporting information}

Supporting informations are available from VBRI Press.

\section{References}

1 Thangaraju, B.; Kaliannan, P.; Semicond Sci Technol, 2000, 15, 542.

DOI: $\underline{10.1088 / 0268-1242 / 15 / 6 / 309 .}$.

2 Szendrei, K.; Gomulya, W.; Yarema, M.; Heiss, W.; Loi, M. A.; Appl. Phys. Lett., 2010, 97,

DOI: $\underline{10.1063 / 1.3518067}$.

3 Shockley, W.; Queisser, H. J.; J. Appl. Phys., 1961, 32, 510. DOI: $10.1063 / 1.1736034$.

4 Güneri, E.; Göde, F.; Çevik, S.; Thin Solid Films, 2015, 589, 578. DOI: $10.1016 /$ j.tsf.2015.06.027.

5 Beddek, L.; Messaoudi, M.; Attaf, N.; Aida, M. S.; Bougdira, J.; J Alloys Compd, 2016, 666, 327. DOI: $10.1016 /$ j.jallcom.2016.01.088.
6 Touati, B.; Gassoumi, A.; Alfaify, S.; Kamoun-Turki, N.; Mater Sci Semicond Process, 2015, 34, 82.

DOI: $\underline{10.1016 / \text { j.mssp.2015.02.020. }}$.

7 Bhunia, H.; Kundu, B.; Chatterjee, S.; Pal, A. J.; J. Mater. Chem. C, 2016, 4, 551.

DOI: $10.1039 / \mathrm{c} 5 \mathrm{tc0} 03959 \mathrm{~b}$

8 Chander, S.; Dhaka, M. S.; Adv. Mater. Lett., 2015, 6, 907. DOI: $10.5185 /$ amlett.2015.5926.

9 Zheng, X.; Gao, F.; Ji, F.; Wu, H.; Zhang, J.; Hu, X.; Xiang, Y.; Mater Lett, 2016, 167, 128.

DOI: $10.1016 /$ j.matlet.2015.12.077.

10 Rajashree, C.; Balu, A. R.; Nagarethinam, V. S.; Surf Eng, 2015, 31, 316. DOI: $10.1179 / 1743294415 Y .0000000014$

11 Palomino-Merino, R.; Portillo-Moreno, O.; Flores-García, J. C.; Hernández-Tecorralco, J.; Martínez-Juárez, J.; Moran-Torres, A.; Rubio-Rosas, E.; Hernández-Téllez, G.; Gutiérrez-Pérez, R.; Chaltel-Lima, L. A.; J. Nanosci. Nanotechnol., 2014, 14, 5408. DOI: $10.1166 /$ jnn.2014.8664.

12 Liang, Z.; Su, M.; Wang, H.; Gong, Y.; Xie, F.; Gong, L.; Meng, H.; Liu, P.; Chen, H.; Xie, W.; Chen, J.; ACS Appl. Mater. Interfaces, 2015, 7, 5830 .

DOI: $\underline{10.1021 / \mathrm{am} 508879 \mathrm{~b}}$.

13 Narchi, P.; Alvarez, J.; Chrétien, P.; Picardi, G.; Cariou, R.; Foldyna, M.; Prod'homme, P.; Kleider, J. -P.; i Cabarrocas, P. R.; Nanoscale Res. Lett., 2016, 11, 1. DOI: $10.1186 / \mathrm{s} 11671-016-1268-1$.

14 Melitz, W.; Shen, J.; Kummel, A. C.; Lee, S.; Surf Sci Rep, 2011, $66,1$. DOI: $\underline{10.1016 / j . s u r f r e p .2010 .10 .001 . ~}$

15 De Wolf, P.; Geva, M.; Hantschel, T.; Vandervorst, W.; Bylsma, R. B.; Appl. Phys. Lett., 1998, 73, 2155. DOI: $10.1063 / 1.122408$.

16 Maknys, K.; Douhéret, O.; Anand, S.; Appl. Phys. Lett., 2003, 83, 2184. DOI: $\underline{10.1063 / 1.1611619}$.

17 Jiang, C. -S.; Moutinho, H. R.; Dhere, R. G.; Al-Jassim, M. M.; IEEE J. Photovoltaics, 2013, 3, 1383. DOI: $10.1109 /$ JPHOTOV.2013.2276932.

18 Jiang, C. -S.; Contreras, M. A.; Mansfield, L. M.; Moutinho, H. R.; Egaas, B.; Ramanathan, K.; Al-Jassim, M. M.; Appl. Phys. Lett., 2015, 106, DOI: $10.1063 / 1.4907165$.

19 Jiang, C. -S, M. A. Contreras, I. L. Repins, L. M. Mansfield, C. Beall, K. Ramanathan, M. M. Al-Jassim, "Nanoscale electrical properties of wide-bandgap $\mathrm{Cu}(\mathrm{In}, \mathrm{Ga}) \mathrm{Se} 2$ and $\mathrm{Cu} 2 \mathrm{ZnSn}(\mathrm{SSe}) 4$ thin films", presented at 40th IEEE Photovoltaic Specialist Conference, PVSC 2014, 8 June 2014 through 13 June 2014, 2014. DOI: $10.1109 /$ PVSC.2014.6925651.

20 Li, H.; Jiang, C.; Metzger W.; Shih, C. -K.; Al-Jassim, M., "Nanometer-scale study of resistance on CdTe solar cell devices", presented at 40th IEEE Photovoltaic Specialist Conference, PVSC 2014, 8 June 2014 through 13 June 2014, 2014. DOI: $10.1109 /$ PVSC.2014.6925267.

21 Zhang, Y.; He, W.; Microelectron. Int., 2012, 29, 35. DOI: $10.1108 / 13565361211219149$.

22 Liu, J.; Mandal, K. C.; Koley, G., "Investigation of CdZnTe crystal defects using scanning spreading resistance microscopy", presented at Hard X-Ray, Gamma-Ray, and Neutron Detector Physics X, San Diego, CA, 11 August 2008 through 13 August 2008, 2008. DOI: $10.1117 / 12.796253$.

23 Ou, X.; Kanungo, P. D.; Kögler, R.; Werner, P.; Gösele, U.; Skorupa, W.; Wang, X.; Nano Lett., 2010, 10, 171. DOI: $10.1021 / \mathrm{n} 1903228 \mathrm{~s}$.

24 Qin, S.; Suo, Z.; Fillmore, D.; Lu, S.; Jeff Hu, Y.; McTeer, A.; Appl. Phys. Lett., 2013, 103, DOI: $10.1063 / 1.4858963$.

25 Wang, L.; Chauveau, J. M.; Brenier, R.; Sallet, V.; Jomard, F.; Sartel, C.; Brémond, G.; Appl. Phys. Lett., 2016, 108, DOI: $10.1063 / 1.4945100$.

26 Zhang, L.; Koike, M.; Ono, M.; Itai, S.; Matsuzawa, K.; Ono, S.; Saito, W.; Yamaguchi, M.; Hayase, Y.; Hara, K.; Microelectron. Reliab., 2015, 55, 1559.

DOI: $\underline{10.1016 / \mathrm{j} . \text { microrel.2015.06.142. }}$. 
27 Yamazaki, T.; Mizutani, U.; Iwama, Y.; Japanese Journal of Applied Physics Part 1-Regular Papers Brief Communications \& Review Papers, 1983, 22, 454.

DOI: $10.1143 / \mathrm{JJAP} .22 .454$.

28 Ajili, M.; Castagné, M.; Turki, N. K.; Optik, 2015, 126, 708. DOI: 10.1016/j.ijleo.2015.02.039.

29 Touati, B.; Gassoumi, A.; Dobryden, I.; Natile, M. M.; Vomiero, A.; Turki, N. K.; Superlattices Microstruct, 2016, 97, 519. DOI: $10.1016 /$ j.spmi.2016.07.025.

30 Hansen, W. N.; Hansen, G. J.; Surf. Sci., 2001, 481, 172. DOI: $10.1016 /$ S0039-6028(01)01036-6.

31 Kumar, R.; Das, R.; Gupta, M.; Ganesan, V.; Superlattices Microstruct, 2014, 75, 601. DOI: $10.1016 /$ j.spmi.2014.08.019.

32 Baumgart, C.; Helm, M.; Schmidt, H.; Phys. Rev. B Condens. Matter Mater. Phys., 2009, 80 , DOI: $10.1103 /$ PhysRevB.80.085305.

33 Zhang, Y.; Pluchery, O.; Caillard, L.; Lamic-Humblot, A. -F.; Casale, S.; Chabal, Y. J.; Salmeron, M.; Nano Lett., 2015, 15, 51. DOI: $\underline{10.1021 / \mathrm{nl} 503782 \mathrm{~s}}$.

34 Melitz, W.; Shen, J.; Lee, S.; Lee, J. S.; Kummel, A. C.; Droopad, R.; Yu, E. T.; J. Appl. Phys., 2010, 108, DOI: $\underline{10.1063 / 1.3462440}$.

35 Li, S.; Zhou, Y.; Zi, Y.; Zhang, G.; Wang, Z. L.; ACS Nano, 2016, 10, 2528. DOI: $10.1021 /$ acsnano.5b07418.

36 Kulis, P.; Butikova, J.; Polyakov, B.; Marcins, G.; Pervenecka, J.; Pudzs, K.; Tale, I., "Work function of colloidal semiconducting nanocrystals measured by Kelvin probe", presented at International Conference on Functional Materials and Nanotechnologies, FM and NT 2012, Riga, 17 April 2012 through 20 April 2012, 2012. DOI: $10.1088 / 1757-899 \mathrm{X} / 38 / 1 / 012048$.

37 Bârsan, O. A.; Hoffmann, G. G.; Van Der Ven, L. G. J.; De With, G.; ACS Appl. Mater. Interfaces, 2016, 8, 19701. DOI: 10.1021 /acsami.6b06201.

38 Guo, D. -Z.; Hou, S. -M.; Zhang, G. -M.; Xue, Z. -Q.; Appl. Surf. Sci., 2006, 252, 5149.

DOI: $10.1016 / \mathrm{j}$.apsusc.2005.07.058.

39 Bietsch, A.; Schneider, M. A.; Welland, M. E.; Michel, B.; J Vac Sci Technol B Microelectron Nanometer Struct, 2000, 18, 1160. DOI: $10.1116 / 1.591353$.

40 Ho, Yeon D.; Chandra Mohanty, B.; Lee, S. M.; Soo Cho, Y.; Sci. Rep., 2015, 5 ,

DOI: $10.1038 /$ srep14353.

41 Lashkova, N. A.; Permiakov, N. V.; Maximov, A. I.; Spivak, Y. M.; Moshnikov, V. A.; St.Petersburg Polytechnical University Journal: Physics and Mathematics, 2015, 1, 15.

DOI: $10.1016 /$ j.spjpm.2015.03.014.

42 Rhoderick, E. H.; IEE Proc Part I, 1982, 129, 1. DOI: $10.1049 / \mathrm{ip}-\mathrm{i}-1.1982 .0001$.

43 Lu, R. P.; Kavanagh, K. L.; Dixon-Warren, S. J.; Spring Thorpe, A. J.; Streater, R.; Calder, I.; J Vac Sci Technol B Microelectron Nanometer Struct, 2002, 20, 1682. DOI: $\underline{10.1116 / 1.1496512 .}$

44 Mishra, U. K.; Singh J. Semiconductor junctions, In Semiconductor Device Physics and Design; Springer: The Netherlands, 2008, pp. 216-245. DOI: $10.1007 / 978-1-4020-6481-4$.

45 Lee, W.; Dasgupta, N. P.; Prinz, F. B., "Scanning tunneling microscopy of quantum confinement effects in lead sulfide thin films", presented at 2009 34th IEEE Photovoltaic Specialists Conference, PVSC 2009, Philadelphia, PA, 7 June 2009 through 12 June 2009, 2009. DOI: $10.1109 /$ PVSC.2009.5411631.

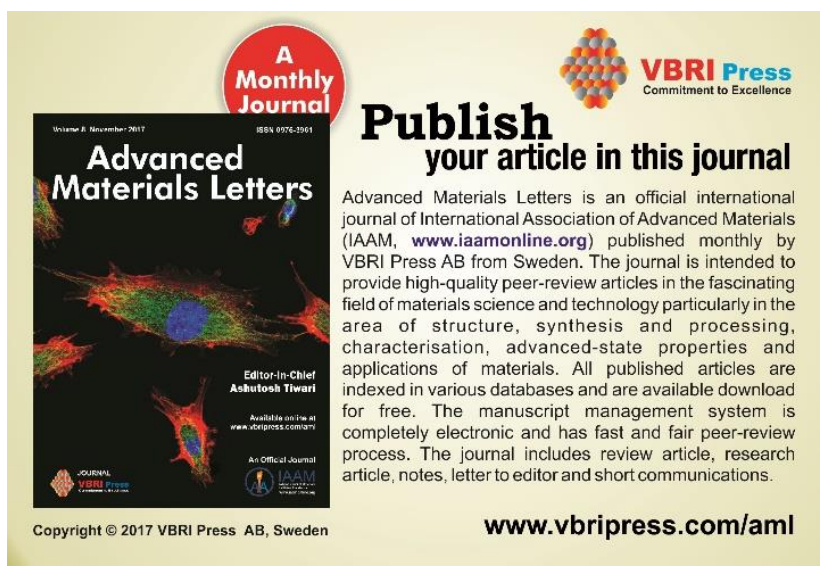




\section{SUPPORTING INFORMATION}
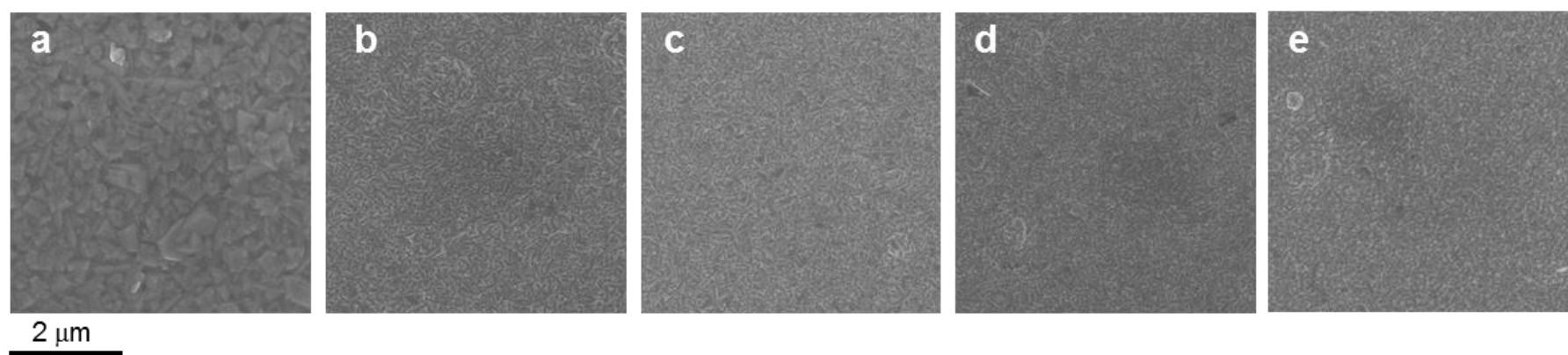

Fig. S1. HRSEM images for the pure and Cu-doped PbS thin films grown by CBD. (a): pure, (b): 0.5 at $\%$, (c): 1.0 at $\%$, (d): 1.5 at $\%$ and (e): 2.0 at $\%$. 